\title{
Real-time Classification of Finger Movements using Two-channel Surface Electromyography
}

\author{
Khairul Anam ${ }^{1,2}$ and Adel Al-jumaily ${ }^{2}$ \\ ${ }^{1}$ University of Jember, Jember, Indonesia \\ ${ }^{2}$ University of Technology, Sydney, Australia \\ khairul.anam@student.uts.edu.au,adel.al-jumaily@uts.edu.au
}

\begin{abstract}
Keywords: $\quad$ Surface EMG, Extreme Learning Machine, Finger Movements.
Abstract: $\quad$ The use of a small number of Electromyography (EMG) channels for classifying the finger movement is a challenging task. This paper proposes the recognition system for decoding the individual and combined finger movements using two channels surface EMG. The proposed system utilizes Spectral Regression Discriminant Analysis (SRDA) for dimensionality reduction, Extreme Learning Machine (ELM) for classification and the majority vote for the classification smoothness. The experimental results show that the proposed system was able to classify ten classes of individual and combined finger movements, offline and online with accuracy $97.96 \%$ and $97.07 \%$ respectively.
\end{abstract}

\section{INTRODUCTION}

The electromyography signal has been used widely to control the upper-limb prosthetic robot to recover the quality of life of the amputee. Many attempts have been made to decode the hand movements as the control sources of the hand robot (Oskoei and Huosheng, 2008): (Sang Wook et al., 2011); (Micera et al., 2010). The dexterous control system should involve not only the hand movements but also the finger movements (Tenore et al., 2009); (Khushaba et al., 2012). Some efforts have been done to recognize the finger movements. Tenore et al decoded ten classes of the individual finger movements by using up to 32 sEMG channels with accuracy $\sim 90 \%$ (Tenore et al., 2009). In addition, Al-Timemy et al (Al-Timemy et al., 2013) classified 15 individual finger movements and achieved $98 \%$ accuracy by using 6 sEMG channels.

The use of few numbers of electrodes in a finger recognition system without compromising the decoding accuracy is a challenging task. Tsenov et al used two sEMG channels for 4 class finger movements i.e. the thumb, index, middle finger and hand closure with the best accuracy was nearly $93 \%$ in offline classification (Tsenov et al., 2006). Moreover, Khusaba et al classified 10 classes of individual and combined finger movements which consisted of five individual finger movements by using two sEMG channels (Khushaba et al., 2012).
This work could achive $92 \%$ and $90 \%$ of accuracy for the offline and online classification respectively.

To achieve good classification results, it demands the proper and right decoding methods. Tsenov employed time domain feature extractions and Artificial Neural Networks (ANNs) to process the sEMG signals from two channels (Tsenov et al., 2006). This recognition system gave a good accuracy in offline classification but no evidence in online classification. In addition, this system only decoded for finger movements which were only three individual finger movements and one hand close. More finger movements are needed in realtime application.

The best improvement was proposed in (Khushaba et al., 2012). The sEMG signals from two channels were extracted by using time domain features and reduced by Linear Discriminant Analysis (LDA) and then classified by using Support Vector Machine. The final results were refined by using a Bayesian fusion vote. Ten classes of individuated and combined finger movements were able to recognize with $92 \%$ offline classification accuracy and 90\% online classification accuracy.

The achievement attained by previous system is good but not good enough for the implementation in real-time application. Many attempts should be made to achieve more accurate system recognition. For that goal, this paper proposes the novel recognition system which uses two sEMG channels 
in recognizing the individual and combined finger movements. A number of features are extracted by using time domain feature extraction and then reduced by using Spectral Regression Discriminant Analysis (SRDA) (Cai et al., 2008). SRDA is an extension of Linear Discriminant Analysis which is fast and able to work on a large dataset.

Extreme Learning Machine (ELM)(Huang et al., 2012) is used for classification. ELM is generalized" single-hidden-layer feedforward networks (SLFNs) whose hidden layer does not need to be tuned. It needs fewer optimization constraint, has better generalization functioning and faster learning time than SVM (Huang et al., 2012). This combination, SRDA and ELM along with the majority vote (Chan and Green, 2007), provide a fast and an accurate classification system for individuated and combined finger movements.

\section{METHOD}

\subsection{Experiment Procedures}

The data in this work were acquired from six subjects, one female and five males. All subjects were normally limbed with no muscle disorder. To avoid the effect of position movement on EMG signals, subject's arm was supported and fixed at certain position as decribed in fig. 2.(Khushaba et al., 2012).

The FlexComp Infiniti ${ }^{\mathrm{TM}}$ System from Thought Technology was used to process the signals from two EMG MyoScan ${ }^{\mathrm{TM}}$ T9503M Sensors which were put on the subject's forearm as seen in the figure 1 . The acquired EMG signals were amplified to a total gain of 1000 and sampled at $2000 \mathrm{~Hz}$.

The collected EMG signals were processed in the Matlab 2012b installed in the Intel Core i5 $3.1 \mathrm{GHz}$ desktop computer with 4 GB RAM running on Windows 7 operating system. The signals were filtered by a band pass filter between 20 and $500 \mathrm{~Hz}$ with a notch filter to remove the $50 \mathrm{~Hz}$ line interference. Finally, the EMG signals were down sampled to $1000 \mathrm{~Hz}$.

Fig. 2 shows ten classes of the individual and combined finger movements consisting of the flexion of individuated fingers, i.e., Thumb (T), Index (I), Middle (M), Ring (R), Little (L) and the pinching of combined Thumb-Index (T-I), ThumbMiddle (T-M), Thumb-Ring (T-R), Thumb-Little (T-L), and the hand close (HC).

The offline classification was performed based on data from the data acquisition. In this stage, the subjects asked to perform a certain posture of a finger movement for a period $5 \mathrm{~s}$ and then take a rest for $5 \mathrm{~s}$. Each movement was repeated six times. Therefore 30 minutes of data are collected for each trials and 180 minutes for all repetitions. The data collected were divided into training data and testing data. Four of six trials were training data and the rest were testing data.

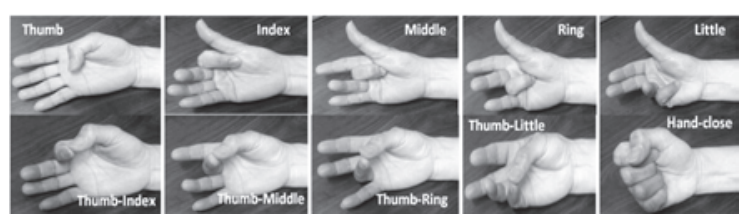

Figure 1: Ten different finger movements.

In the online stage, the subject performed similar activities. The difference is the repetition which is only four times instead of six and all are for testing only. Another difference is the recognition system is performed each $100 \mathrm{~ms}$ and then the result is displayed on the screen.

\subsection{Proposed Method}

The proposed recognition system consisted of two stages, an offline and online classification stages. In the offline stage, the EMG signals were acquired by a data acquisition device from 6 subjects. The filtering and windowing was applied to the collected data before being extracted by using a time domain feature set. To reduce the dimension of the features, SRDA was employed. Then, the reduced data were classified using ELM and refined by using the majority vote. The trained ELM which is produced by the offline classification is stored and used in the online classification stage.

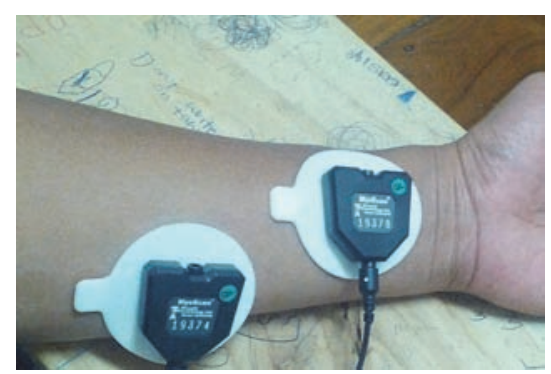

Figure 2: The electrodes placement.

In the online stage, the trained ELM is restored and used to classify the sEMG signals which are captured every $100 \mathrm{~ms}$. The acquired signals are extracted by using time domain feature extractions and reduced their dimensionality by using SRDA. 
Then, the reduced features are recognized by the trained ELM and the output classification is refined by using majority vote.

\subsection{Feature Extraction}

The features were extracted from a time domain feature set which consists of Waveform Length (WL), Slope Sign Changes (SSC), Number of Zero Crossings (ZCC), and Sample Skewness (SS). In addition, some parameters from Hjorth Time Domain Parameters (HTD) and Auto Regressive (AR) Model Parameters were included as used in (Khushaba et al., 2012). All features were extracted by using myolectric toolbox (Chan and Green, 2007) and Biosig toolbox (Schlogl and Brunner, 2008).

The AR model parameters have been proven to be stable and robust to the electrode location shift and the change of signal level (Tkach et al., 2010). Moreover, aforementioned time domain features were windowed by using disjoint window instead of sliding window to keep computational cost low. A $100 \mathrm{~ms}$ window and a 100 increments were used to form a system which is suitable for real time application.

\subsection{SRDA}

SRDA is an improvement of LDA which is better than LDA in the computational aspect and the ability to cope with a large dataset (Cai et al., 2008). Let eigen problem of LDA is

$$
\bar{X} W \bar{X}^{T} a=\lambda \bar{X} \bar{X}^{T} a
$$

where $\bar{X}\left(\begin{array}{lll}1 & \mathrm{x} & \mathrm{c}\end{array}\right)$ is centered data matrix, $W$ is eigenvector matrix $(m \times m), \lambda=$ eigenvalue, $\mathrm{a}=$ transformation vector, $\mathrm{c}=$ the number of classes, and $\mathrm{m}=$ the number of total training data points. Modification of the equation (1) gives:

$$
W \bar{y}=\lambda \bar{y}
$$

where

$$
\bar{X}^{T} a=\bar{y}
$$

The solution of LDA problem by SRDA is to get $y$ by solving eq (2) and then use the y obtained to find $a$. To solve $a$, the least square sense could be employed by using:

$$
a=\underset{a}{\arg \min } \sum_{i=1}^{m}\left(a^{T} \bar{x}_{i}-\bar{y}_{i}\right)^{2}
$$

Regularize least square problem of SRDA, we get:

$$
a=\underset{a}{\arg \min } \sum_{i=1}^{m}\left(\left(\bar{X}^{T} a-\bar{y}\right)^{T}\left(\bar{X}^{T} a-\bar{y}\right)+\alpha a^{T} a\right)
$$

Derivative of equation (5) gives:

$$
\begin{gathered}
\left(\bar{X} \bar{X}^{T}+\alpha I\right)=\bar{X} \bar{y} \\
\Rightarrow a=\left(\bar{X} \bar{X}^{T}+\alpha I\right)^{-1} \bar{X} \bar{y}
\end{gathered}
$$

\subsection{Extreme Learning Machine}

ELM is a learning scheme for single layer feedforward networks (SLFNs). While the network parameters are tuned in classical SLFNs learning algorithms, most of these parameters are analytically determined in ELM. The hidden parameters can be independently determined from the training data, and the output parameters can be determined by pseudo-inverse method using the training data. As a result, the learning of ELM can be carried out extremely fast compared to the other learning algorithms (Huang et al., 2012).

The output function of ELM for generalized SLFNs (for one output node case) is:

$$
f_{L}(x)=\sum_{i=1}^{L} \beta_{i} h_{i}(x)=\mathbf{h}(\mathbf{x}) \boldsymbol{\beta}
$$

where $\boldsymbol{\beta}=\left[\beta_{1}, \ldots, \beta_{L}\right]^{T}$ is the vector of the output weight between hidden layer of $\mathrm{L}$ nodes and the output node, $\mathbf{h}(\mathbf{x})=\left[h_{1}(x), \ldots, h_{L}(x)\right]$ is the output vector of hidden layer.

The objective of ELM is to minimize the error and the norm of weight:

$$
\text { Minimize : }\|\mathbf{H} \boldsymbol{\beta}-\mathbf{T}\|^{2} \text { and }\|\boldsymbol{\beta}\|
$$

where $\mathbf{T}$ is the target. For classification purpose, the output function of ELM in equation (7) could be modified to be:

$$
\mathrm{f}(\mathrm{x})=\mathbf{h}(\mathbf{x}) \boldsymbol{\beta}=\mathbf{h}(\mathbf{x}) \mathbf{H}^{\mathrm{T}}\left(\frac{1}{\mathrm{C}}+\mathbf{H} \mathbf{H}^{\mathrm{T}}\right)^{-1} \mathrm{~T}
$$

where

$$
\mathbf{H}=\left[\begin{array}{c}
\mathbf{h}\left(\mathrm{x}_{1}\right) \\
\vdots \\
\mathbf{h}\left(\mathrm{x}_{\mathrm{N}}\right)
\end{array}\right]=\left[\begin{array}{ccc}
h_{1}\left(\mathrm{x}_{1}\right) & \cdots & h_{L}\left(\mathrm{x}_{1}\right) \\
\vdots & \vdots & \vdots \\
h_{1}\left(\mathrm{x}_{\mathrm{N}}\right) & \cdots & h_{L}\left(\mathrm{x}_{\mathrm{N}}\right)
\end{array}\right]
$$

as well as $\mathrm{C}$ is a user-specified parameter and $\mathrm{N}$ is the number of the training data. In the equation (10), 
$h(x)$ is a feature mapping (hidden layer output vector) which can be:

$$
\mathrm{h}(\mathrm{x})=\left[G\left(a_{1}, b_{1}, \mathrm{x}\right), \ldots, G\left(a_{L}, b_{L}, \mathrm{x}\right)\right]
$$

where $G$ is a non-linear piecewise continuous function such as sigmoid, hard limit, Gaussian, and multi quadratic function.

If the feature mapping $h(x)$ is unknown to the user, a kernel function can be used to represent $\mathrm{h}(\mathrm{x})$. Then, the equation (9) would be:

$$
\begin{aligned}
\mathrm{f}(\mathrm{x}) & =\mathbf{h}(\mathbf{x}) \mathbf{H}^{\mathrm{T}}\left(\frac{1}{\mathrm{C}}+\mathbf{H H}^{\mathrm{T}}\right)^{-1} \mathrm{~T} \\
& =\left[\begin{array}{c}
K\left(\mathrm{x}, \mathrm{x}_{1}\right) \\
\vdots \\
K\left(\mathrm{x}, \mathrm{x}_{\mathrm{N}}\right)
\end{array}\right]\left(\frac{1}{\mathrm{C}}+\Omega_{\mathrm{ELM}}\right)^{-1} \mathrm{~T}
\end{aligned}
$$

where

$$
\Omega_{\mathrm{ELM}}=\mathbf{H H}^{\mathrm{T}}: \Omega_{\mathrm{ELMi}, j}=h\left(\mathrm{x}_{i}\right) \cdot h\left(\mathrm{x}_{j}\right)=K\left(\mathrm{x}_{i}, \mathrm{x}_{j}\right)
$$

and $\mathrm{K}$ is a kernel function such that :

$$
K(\mathbf{u}, \mathbf{v})=\exp \left(-\gamma\|\mathbf{u}-\mathbf{v}\|^{2}\right)
$$

\subsection{Majority Vote}

The majority vote was used to refine the classification results. It utilizes the results from the present state and $n$ previous states and makes a new classification result based on the class which appears most frequent. This procedure produces the finger movement class that removes specious misclassification. Besides majority vote, the transition states in the classification results are removed too. This method gives the recognition system that works in steady state only regardless the transition state.

\section{RESULT AND DISCUSSION}

The two experiments have been performed, the offline and online classification. In the offline stage, the possibility of adding new channel which was extracted from summing up of two original channels is verified. Next, the best result of the offline stage was utilized in the online classification stage. In the both offline and online stage, the signals were extracted from six subjects with $100 \mathrm{~ms}$ windows length and100 increment as recommended in
(Khushaba et al., 2012). In addition, the Gaussian kernel based ELM is used as the classifier. It has two importance parameters, $\mathrm{C}$ and $\gamma$ as showed in equation 9 and 12. This paper used the optimized ELM presented in the (Anam et al., 2013) with the $\gamma=2^{-5}$ and $C=2^{0}$. The majority vote method with 9 decision voting was employed to refine the classification result.

The first experiment was the offline classification. In this stage, the performance of the classification system using only two original signals (ch1, ch2) was compared to the two signals plus the new additional channel from summing up of the both channels (ch1, ch2, ch1+ch2). From six trials across each subject, four trials were used to train the ELM and the rest were the testing data. The classification result is shown in the table 1 .

Table 1: The classification results averaged for six subjects.

\begin{tabular}{|c|c|c|}
\hline Subject & Ch1 \& Ch2 (\%) & Ch1, Ch2, Ch1+Ch2 (\%) \\
\hline 1 & $98.48 \pm 2.87$ & $97.10 \pm 4.13$ \\
\hline 2 & $100.00 \pm 0$ & $100.00 \pm 0$ \\
\hline 3 & $94.95 \pm 11.38$ & $96.42 \pm 8.26$ \\
\hline 4 & $98.61 \pm 3.93$ & $98.34 \pm 4.02$ \\
\hline 5 & $98.89 \pm 2.43$ & $98.89 \pm 3.51$ \\
\hline 6 & $93.81 \pm 8.39$ & $96.99 \pm 5.49$ \\
\hline Average & $97.46 \pm 2.35$ & $97.96 \pm 1.47$ \\
\hline
\end{tabular}

Table 1 shows that both configurations achieved good accuracies across six subjects. However, the additional signal of the summation of two channels gave better average accuracy than two channels only even though the difference is not so significant. The significance of the second configuration is depicted in figure 3. Even though both configurations achieve similar accuracy in recognizing the ten finger movements, the standard deviation of second one is better than first one.

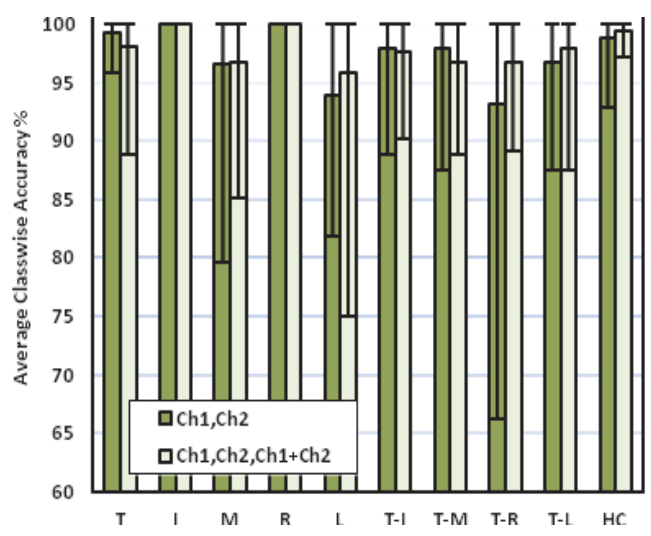

Figure 3: The Average class-wise accuracy in the offline classification. 


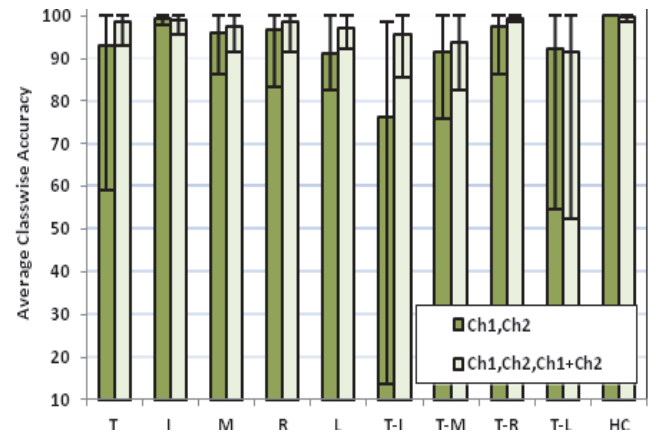

Figure 4: The online classification accuracy.

The online classification is the second experiments performed. The individual and combined finger movements were recognized in real-time based on the matrix projection of SRDA and the trained ELM kernel from offline stage. In this experiments, the configuration of (ch1, ch2) achieve $93.36 \%$ accuracy while the (ch1,ch2, ch1+ch2) configuration attained better accuracy which is $97.07 \%$. The performance of finger recognition is depicted in the fig. 4 and the table 2.

Table 2: The confusion matrix of the classification results averaged for SIX subjects.

\begin{tabular}{|c|c|c|c|c|c|c|c|c|c|c|c|}
\hline & \multicolumn{11}{|c|}{ Intended task (\%) } \\
\hline & & $\mathrm{T}$ & I & $\mathrm{M}$ & $\mathrm{R}$ & L & T-I & T-M & T-R & \begin{tabular}{|l|} 
T-L \\
\end{tabular} & HC \\
\hline \multirow{10}{*}{ 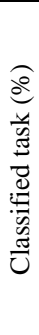 } & $\mathrm{T}$ & \begin{tabular}{|l|l}
98.7 \\
\end{tabular} & 0.1 & 0.0 & 0.0 & 0.0 & \begin{tabular}{|l|l}
0.9 \\
\end{tabular} & 0.0 & \begin{tabular}{|l|l}
0.0 \\
\end{tabular} & \begin{tabular}{|l|l}
0.0 \\
\end{tabular} & \begin{tabular}{|l|l}
0.3 \\
\end{tabular} \\
\hline & I & 0.0 & 99.3 & 0.0 & 0.0 & \begin{tabular}{|l|}
0.0 \\
\end{tabular} & 0.3 & 0.0 & 0.0 & \begin{tabular}{|l|l|}
0.4 \\
\end{tabular} & \begin{tabular}{|l|l|}
0.0 \\
\end{tabular} \\
\hline & $\mathrm{M}$ & 0.0 & 0.0 & 98.0 & \begin{tabular}{|l|l}
0.2 \\
\end{tabular} & 0.0 & \begin{tabular}{|l|}
0.0 \\
\end{tabular} & 1.3 & \begin{tabular}{|l|}
0.0 \\
\end{tabular} & \begin{tabular}{|l|l|}
0.0 \\
\end{tabular} & \begin{tabular}{|l|}
0.5 \\
\end{tabular} \\
\hline & $\mathrm{R}$ & 0.0 & 0.0 & 0.0 & 99.9 & 0.0 & 0.0 & 0.0 & \begin{tabular}{|l|l}
0.1 \\
\end{tabular} & 0.0 & \begin{tabular}{|l|l}
0.0 \\
\end{tabular} \\
\hline & $\mathrm{L}$ & 1.1 & 0.0 & 0.0 & 0.0 & 97.2 & \begin{tabular}{|l|l}
0.6 \\
\end{tabular} & 0.0 & \begin{tabular}{|l|l}
0.0 \\
\end{tabular} & \begin{tabular}{|l|l}
0.1 \\
\end{tabular} & 1.0 \\
\hline & T-I & 0.5 & 0.0 & 0.0 & 0.0 & $\begin{array}{ll}1.5 \\
\end{array}$ & 95.1 & 1.0 & \begin{tabular}{|l|}
0.0 \\
\end{tabular} & \begin{tabular}{|l|l}
1.9 \\
\end{tabular} & \begin{tabular}{|l|}
0.0 \\
\end{tabular} \\
\hline & T-M & 0.0 & 0.0 & 0.9 & 0.0 & 0.7 & \begin{tabular}{|l}
1.3 \\
\end{tabular} & 96.1 & \begin{tabular}{|l|}
0.0 \\
\end{tabular} & \begin{tabular}{|l|l|}
0.1 \\
\end{tabular} & \begin{tabular}{|l|l}
1.0 \\
\end{tabular} \\
\hline & T-R & 0.0 & 0.0 & 0.0 & 0.0 & 0.0 & 0.3 & 0.5 & 99.1 & \begin{tabular}{|l|l|}
0.0 \\
\end{tabular} & 0.0 \\
\hline & T-L & 0.0 & 0.1 & 0.0 & 0.0 & 6.0 & \begin{tabular}{|l|}
0.3 \\
\end{tabular} & 0.7 & \begin{tabular}{|l|}
0.5 \\
\end{tabular} & 92.3 & \begin{tabular}{|l|}
0.0 \\
\end{tabular} \\
\hline & $\mathrm{HC}$ & 0.0 & 0.0 & 0.0 & 0.0 & 0.0 & \begin{tabular}{|l|}
0.0 \\
\end{tabular} & 0.0 & \begin{tabular}{ll|}
0.2 \\
\end{tabular} & \begin{tabular}{|l|}
0.0 \\
\end{tabular} & 99.8 \\
\hline
\end{tabular}

Figure 4 shows that the T-L movement is the most difficult one to recognize. It was misclassified to the $\mathrm{L}$ movements as seen in the confusion matrix table 2. It was probably caused by the facts that the T-L was composed of Thumb(T) and Little(L) finger movement therefore there is possibility each movement affects the combined movements.

Besides the classification performance, the processing time of the real-time application has been also tested which the result is presented in table 3 . The acquisition, filtering, feature extraction and reduction, ELM and majority vote processing time were record during the experiment. This recognition system took $112.13 \mathrm{~ms}$ in average. It is verified that processing time of this system is in between the optimal processing time for real-time myoelectric control, 100-125 ms, as suggested in (Farrell and Weir, 2007).
Table 3: The processing time of the online experiment.

\begin{tabular}{|c|c|c|c|c|c|c|}
\hline \multicolumn{7}{|c|}{ Processing time (ms) } \\
\hline Class & Acquiring & Filter & $\begin{array}{c}\text { Extraction } \\
\text { /reduction }\end{array}$ & ELM & Vote & Total \\
\hline T & 100 & 3.9 & 7.6 & 0.5 & 0.1 & 112.1 \\
\hline I & 100 & 3.5 & 7.2 & 0.5 & 0.1 & 111.3 \\
\hline M & 100 & 3.5 & 7.3 & 0.5 & 0.1 & 111.4 \\
\hline R & 100 & 3.6 & 7.4 & 0.5 & 0.1 & 111.6 \\
\hline L & 100 & 3.7 & 7.6 & 0.6 & 0.1 & 111.9 \\
\hline T-I & 100 & 3.5 & 7.3 & 0.5 & 0.1 & 111.4 \\
\hline T-M & 100 & 3.6 & 7.5 & 0.5 & 0.1 & 111.7 \\
\hline T-R & 100 & 3.6 & 7.6 & 0.6 & 0.1 & 111.8 \\
\hline T-L & 100 & 3.5 & 7.3 & 0.5 & 0.1 & 111.4 \\
\hline HC & 100 & 3.5 & 7.3 & 0.5 & 0.1 & 111.4 \\
\hline Avg & 100 & 3.6 & 7.4 & 0.5 & 0.1 & 112.1 \\
\hline
\end{tabular}

This promising result could be implemented to the hand exoskeleton to recover the motor function of the patients post stroke. It could move all individual fingers and some combined movements. However, it is aimed for finger extension only. In addition, it would not work properly if the EMG signal of the subject is very weak. Therefore, it could be only applied to the partially paralyzed subject.

Furthermore the proposed system could be implemented to the prosthetic hand device. It is promising because it used few electrodes which enhance the user's comfort. However, it needs more validation for amputee subjects.

\section{CONCLUSIONS}

The two channel sEMG signals were used in this paper to recognize the ten individual and combined finger movements. The extracting more feature from summation of the signals from the two channels improves the classification accuracy in both offline and online classification system. By using this combination, the recognition system was able to achieve in average $97.96 \%$ in offline and $97.07 \%$ in online one. These results show the feasibility of the proposed system in classifying ten different finger movements.

\section{REFERENCES}

Al-Timemy, A. H., Bugmann, G., Escudero, J. \& Outram, N. 2013. Classification of Finger Movements for the Dexterous Hand Prosthesis Control with Surface Electromyography. Biomedical and Health Informatics, IEEE Journal of, 17, 608-618.

Anam, K., Khushaba, R. \& Al-Jumaily, A. 2013. TwoChannel Surface Electromyography for Individual and 
Combined Fingers Movements. Accepted paper in 2013 Annual International Conference of the IEEE Engineering in Medicine and Biology Society,EMBC. Osaka.

Cai, D., He, X. \& Han, J. 2008. SRDA: An efficient algorithm for large-scale discriminant analysis. IEEE Transactions on Knowledge and Data Engineering, 20, 1-12.

Chan, A. D. C. \& Green, G. C. Myoelectric control development toolbox. Proceedings of 30th Conference of the Canadian Medical \& Biological Engineering Society, 2007. M0100-1.

Farrell, T. R. \& Weir, R. F. 2007. The Optimal Controller Delay for Myoelectric Prostheses. Neural Systems and Rehabilitation Engineering, IEEE Transactions on, 15, 111-118.

Huang, G. B., Zhou, H., Ding, X. \& Zhang, R. 2012. Extreme learning machine for regression and multiclass classification. IEEE Transactions on Systems, Man, and Cybernetics, Part B: Cybernetics, 42, 513-529.

Khushaba, R. N., Kodagoda, S., Takruri, M. \& Dissanayake, G. 2012. Toward improved control of prosthetic fingers using surface electromyogram (EMG) signals. Expert Systems with Applications.

Micera, S., Carpaneto, J. \& Raspopovic, S. 2010. Control of Hand Prostheses Using Peripheral Information. IEEE Reviews in Biomedical Engineering, 3, 48-68.

Oskoei, M. A. \& Huosheng, H. 2008. Support Vector Machine-Based Classification Scheme for Myoelectric Control Applied to Upper Limb. IEEE Transactions on Biomedical Engineering, 55, 1956-1965.

Sang Wook, L., Wilson, K. M., Lock, B. A. \& Kamper, D. G. 2011. Subject-Specific Myoelectric Pattern Classification of Functional Hand Movements for Stroke Survivors. IEEE Transactions on Neural Systems and Rehabilitation Engineering, 19, 558-566.

Schlogl, A. \& Brunner, C. 2008. BioSig: a free and open source software library for BCI research. Computer, 41, 44-50.

Tenore, F. V. G., Ramos, A., Fahmy, A., Acharya, S., Etienne-Cummings, R. \& Thakor, N. V. 2009. Decoding of individuated finger movements using surface electromyography. IEEE Transactions on Biomedical Engineering, 56, 1427-1434.

Tkach, D., Huang, H. \& Kuiken, T. A. 2010. Study of stability of time-domain features for electromyographic pattern recognition. Journal of NeuroEngineering and Rehabilitation, 7, 21.

Tsenov, G., Zeghbib, A. H., Palis, F., Shoylev, N. \& Mladenov, V. Neural Networks for Online Classification of Hand and Finger Movements Using Surface EMG signals. 8th Seminar on Neural Network Applications in Electrical Engineering (NEUREL), 2527 Sept. 2006 2006. 167-171. 


\section{NEUROTECHNIX 2013}

International Congress on Neurotechnology, Electronics and Informatics

VILAMOURA, Algarve, Portugal

18 - 20 September, 2013

\section{PROCEEDINGS}

SPONSORED BY:

IITSTICC () MedlnRes
IN COOPERATION WITH:

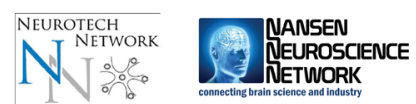

APezenc
IN COLLABORATION WITH:

antibodies 


\title{
NEUROTECHNIX
}

\section{3}

\author{
Proceedings of the \\ International Congress on Neurotechnology, \\ Electronics and Informatics
}

Vilamoura, Algarve, Portugal

18 - 20 September, 2013

Sponsored by

INSTICC - Institute for Systems and Technologies of Information, Control and Communication

In Cooperation with

Neurotech Network

Nansen Neuroscience Network

APEEGNC - Associação Portuguesa de EEG e Neurofisiologia Clínica SPN - Sociedade Portuguesa de Neurologia

Enlightenment FP7

In Collaboration with

antibodies-online.com - The Marketplace for Research Antibodies 
Copyright (C) 2013 SCITEPRESS - Science and Technology Publications All rights reserved

Edited by Ana Rita Londral, Pedro Encarnação and Jose Luis Pons

Printed in Portugal

ISBN: 978-989-8565-80-8

Depósito Legal: 363650/13

http://www.neurotechnix.org

neurotechnix.secretariat@insticc.org 


\section{BRIEF CONTENTS}

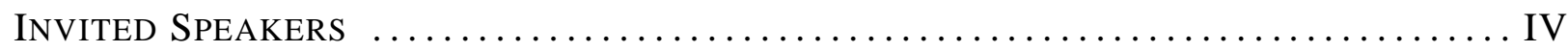

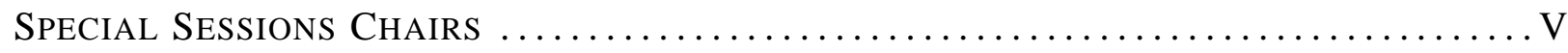

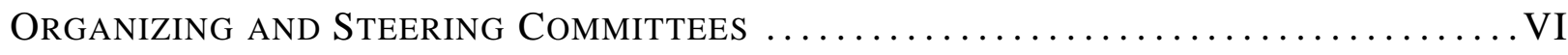

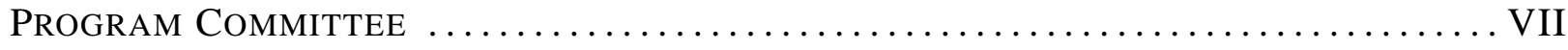

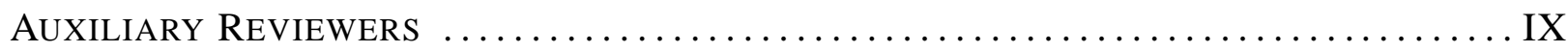

Special Sessions Program Committee $\ldots \ldots \ldots \ldots \ldots \ldots \ldots \ldots \ldots \ldots \ldots \ldots \ldots \ldots \ldots$

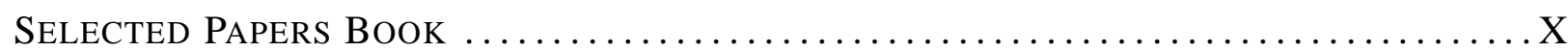

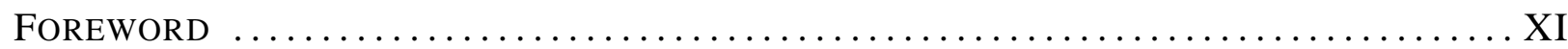

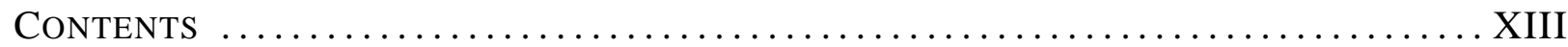




\title{
INVITED SPEAKERS
}

\author{
Kevin Warwick \\ University of Reading \\ U.K.
}

François Hug

The University of Queensland

Australia

Aldo Faisal

Imperial College London

U.K.

\section{Alexandre Castro-Caldas}

Portuguese Catholic University

Portugal 


\section{SPECial SeSSions Chairs}

\section{Special Session on Virtual and Augmented Reality Systems for UpPer Limbs REHABILITATION}

Alessandro de Mauro, Vicomtech-IK4, Spain

Iris Dimbwadyo Terrer, National Spinal Cord Injury Hospital - SESCAM, Spain

Gorka Epelde, Vicomtech-IK4, Applications for Independent living Group, Spain

\section{Special Session on Decoding the Neural Drive to Muscle through the Analysis of Motor} NEURON SPIKE TRAINS

Juan Álvaro Gallego, Spanish National Research Council (CSIC), Spain

Jose Luis Pons, Instituto de Automatica Industrial, Spain

\section{SPECIAL SESSion ON SENSORY FuSiOn FOR Diagnostics AND NEUROREHABILITATION}

Diego Torricelli, Consejo Superior de Investigaciones Cientificas (CSIC), Spain

Rafael Raya, Spanish National Council for Science Research, Spain

\section{Special Session on Wearable Robotics for Motion Assistance and Rehabilitation}

Nicola Vitiello, Scuola Superiore Sant'Anna, Italy

Samer Mohammed, Lissi - Université Paris-Est Créteil, France

Juan Moreno, CSIC, Spain

\section{SPECIAL SESSION ON BRAIN-COMPUTER INTERFACES AND BRAIN STIMULATION FOR NEUROREHABILITATION}

Martin Bogdan, Universität Leipzig, Germany

Ander Ramos-Murguialday, Eberhard-Karls-University, Germany

Armin Walter, Eberhard-Karls-University Tübingen, Germany

Francisco Perales, Uib, Spain 


\section{Organizing And Steering Committees}

\section{Conference Chair}

Ana Rita Londral, Universidade de Lisboa, Portugal

\section{Program Co-chairs}

Pedro Encarnação, Universidade Católica Portuguesa, Portugal Jose Luis Pons, Instituto de Automatica Industrial, Spain

\section{Proceedings Production}

Marina Carvalho, INSTICC, Portugal

Helder Coelhas, INSTICC, Portugal

Bruno Encarnação, INSTICC, Portugal

Ana Guerreiro, INSTICC, Portugal

Andreia Moita, INSTICC, Portugal

Raquel Pedrosa, INSTICC, Portugal

Vitor Pedrosa, INSTICC, Portugal

Sara Santiago, INSTICC, Portugal

José Varela, INSTICC, Portugal

\section{CD-ROM PRODUCTION}

Pedro Varela, INSTICC, Portugal

\section{Graphics Production and Webdesigner}

André Lista, INSTICC, Portugal

Mara Silva, INSTICC, Portugal

SeCRETARIAT

Cláudia Pinto, INSTICC, Portugal

\section{WEBMASTER}

Susana Ribeiro, INSTICC, Portugal 


\section{Program Committee}

Amr Abdel-Dayem, Laurentian University, Canada

Gregor Adriany, University of Minnesota, U.S.A.

Lyuba Alboul, Sheffield Hallam University, U.K.

Pedro Almeida, University of Lisbon, Portugal

Alexandre Andrade, Faculdade de Ciências da Universidade de Lisboa, Portugal

Helder Araújo, University of Coimbra, Portugal

Sabri Arik, Istanbul University, Turkey

Tetsuya Asai, Hokkaido University, Japan

Jose M. Azorin, Miguel Hernandez University, Spain

Reneta Barneva, State University of New York, U.S.A.

Ammar Belatreche, University of Ulster, U.K.

Alexandre Bernardino, Instituto Superior Técnico, Portugal

Fernando Brunetti, Consejo Superior de Investigaciones Científicas, Spain

Barbara Caputo, IDIAP Research Institute, Switzerland

Xin Chen, Georgia Health Sciences University, U.S.A.

Albert C. S. Chung, The Hong Kong University of Science and Technology, Hong Kong

Emmanuel Conchon, University of Toulouse, IRIT/ISIS, France

Lei Ding, University of Oklahoma, U.S.A.

Dominique Durand, Case Western Reserve, U.S.A.

Jimmy T. Efird, East Carolina Heart Institute, U.S.A.

Gary Egan, Monash University, Australia

Patrícia Figueiredo, Instituto Superior Técnico, Portugal

Alexander Fingelkurts, BM-Science - Brain \& Mind Technologies Research Centre, Finland
Andrew Fingelkurts, BM-Science - Brain \& Mind Technologies Research Centre, Finland

Anselmo Frizera, UFES, Brazil

Colin Fyfe, University of the West of Scotland, U.K.

Vasco Galhardo, Faculdade de Medicina Universidade do Porto, Portugal

Petia Georgieva, University of Aveiro, Portugal

Michele Giugliano, University of Antwerp, Belgium

Jordi Gonzàlez, Universitat Autònoma de Barcelona - Centre de Visió per Computador, Spain

Jay Gunkelman, Brain Science International, U.S.A.

Chung Y. Hsu, China Medical University, Taiwan

Xavier Intes, Rensselaer Polytechnic Institute, U.S.A.

Pasi Karjalainen, University of Eastern Finland, Finland

Jonghwa Kim, University of Augsburg, Germany

Frank Kirchner, DFKI, Germany

Andrzej Kloczkowski, Ohio State University, U.S.A.

Constantine Kotropoulos, Aristotle University of Thessaloniki, Greece

Ondrej Krejcar, University of Hradec Kralove, Czech Republic

Hongen Liao, The University of Tokyo, Japan

Diego Liberati, National Research Council, Italy

Xiao Liu, Brunel University, U.K.

Christos Loizou, Intercollege, Cyprus

Ana Rita Londral, Universidade de Lisboa, Portugal

Nigel Lovell, University of New South Wales, Australia

Mai S. Mabrouk, Misr University for Science and Technology, Egypt

Alessandro de Mauro, Vicomtech-IK4, Spain 


\section{Program Committee (CONT.)}

Javier Melenchón, Universitat Oberta de Catalunya, Spain

Paulo Mendes, University of Minho, Portugal

Susana Novais, Carnegie Mellon University, U.S.A.

Calogero Maria Oddo, Scuola Superiore Sant'Anna, Italy

Haluk Ogmen, University of Houston, U.S.A.

Joao Papa, Sao Paulo State University - Unesp, Brazil

Francisco Perales, Uib, Spain

George Perry, University of Texas at San Antonio, U.S.A.

Victor Pikov, Huntington Medical Research Institutes, U.S.A.

Armando J. Pinho, University of Aveiro, Portugal

Susana Pinto, Institute of Molecular Medicine Faculty of Medicine, University of Lisbon, Portugal

Gabriel Pires, Institute for Systems and Robotics Coimbra / Polytechnic Institute of Tomar, Portugal

Hemerson Pistori, Dom Bosco Catholic University, Brazil

Mirjana Popovic, University of Belgrade, Serbia

Ales Prochazka, Institute of Chemical Technology, Czech Republic

Rafael Raya, Spanish National Council for Science Research, Spain
Despina Sanoudou, Medical School, University of Athens, Greece

André Saúde, Federal University of Lavras, Brazil

Friedhelm Schwenker, University of Ulm, Germany

Mário Forjaz Secca, CEFITEC, Departamento de Fisica, FCT/UNL, Portugal

Tapio Seppänen, University of Oulu, Finland

Miguel Tavares da Silva, Instituto Superior Técnico, Portugal

Claudia Sommer, University of Würzburg, Germany

Armando J. Sousa, Faculdade de Engenharia da Universidade do Porto, Portugal

Sundaram Suresh, Nanyang Technological University, Singapore

João Manuel R. S. Tavares, FEUP - Faculdade de Engenharia da Universidade do Porto, Portugal

Marc Tittgemeyer, Max-Planck-Institut für neurologische Forschung, Germany

Diego Torricelli, Consejo Superior de Investigaciones Cientificas (CSIC), Spain

Carlos M. Travieso, University of Las Palmas de Gran Canaria, Spain

Nicola Vitiello, Scuola Superiore Sant'Anna, Italy

Zeyun Yu, University of Wisconsin at Milwaukee, U.S.A.

Inga Zerr, University of Göttingen, Germany

Djemel Ziou, Universite de Sherbrooke, Canada

\section{AuXiliary Reviewers}

Maria Laura Blefari, EPFL, Switzerland

Henrik Jörntell, Lund University, Sweden

\author{
Aikaterini Koutsou, CAR-CSIC, Spain \\ Cristina Santos, University of Minho, Portugal \\ Shingo Shimoda, RIKEN, Japan
}




\section{Special Sessions Program Committee}

\section{SPECIAL Session on Virtual AND Augmented REAlity Systems for UPPER LIMBS REHABILITATION}

Gorka Epelde, Vicomtech-IK4, Applications for Independent living Group, Spain

Nestor Garay-Vitoria, University of the Basque Country/Euskal herriko Unibertsitatea, Spain
Alessandro de Mauro, Vicomtech-IK4, Spain

Iris Dimbwadyo Terrer, National Spinal Cord Injury Hospital - SESCAM, Spain

\section{Special Session on Decoding the Neural Drive to Muscle through the Analysis of Motor Neuron Spike Trains}

Jakob Lund Dideriksen, Göttingen University, Germany

Aleš Holobar, University of Maribor, Slovenia

\section{SPECIAL SESSION ON SENSORY FUSION FOR DiAgNOSTICS AND NEUROREHABILITATION}

Rafael Raya, Spanish National Council for Science Research, Spain

\section{Special Session on Wearable Robotics for Motion Assistance And REHABILITATION}

Luka Ambrozic, Faculty of Electrical Engineering, University of Ljubljana, Slovenia

Alícia Casals, Institute for Bioengineering of Catalonia.IBEC and Universitat Politècnica de Catalunya.UPC, Spain

Marco Cempini, The Biorobotics Institute - Scuola Superiore Sant'Anna, Pisa, Italy

Mario Cortese, The Biorobotics Institute - Scuola Superiore Sant'Anna, Pisa, Italy

Simona Crea, The BioRobotics Institute - Scuola Superiore Sant'Anna, Pisa, Italy

Antonio J. del-Ama, National Hospital for Spinal Cord Injury, Spain

Georges Fried, Lissi - UPEC, France

Francesco Giovacchini, The Biorobotics Institute Scuola Superiore Sant'Anna, Pisa, Italy
Jian Huang, Huazhong University of Science and Technology, China

Ning Jiang, Universitätsmedizin Göttingen Georg-August-Universität, Germany

Kyoungchul Kong, Sogang University, Korea, Republic of

Stefan Lambrecht, CSIC, Spain

Samer Mohammed, Lissi - Université Paris-Est Créteil, France

Luis Montano, Universidad de Zaragoza, Spain

Juan Moreno, CSIC, Spain

Sehoon Oh, Sogang University, Korea, Republic of Hala Rifai, Lissi - Université Paris-Est Créteil, France

Jan Veneman, Tecnalia, Spain

Nicola Vitiello, Scuola Superiore Sant'Anna, Italy 


\section{Special Sessions Program Committee}

\section{SPECIAL SESSION ON BRAIN-COMPUTER INTERFACES AND BRAIN STIMULATION FOR NEUROREHABILITATION}

Martin Bogdan, Universität Leipzig, Germany

Francisco Perales, Uib, Spain
Ander

Ramos-Murguialday,

Eberhard-Karls-University, Germany

Armin Walter, Eberhard-Karls-University Tübingen, Germany

\section{Selected Papers Book}

A number of selected papers presented at NEUROTECHNIX 2013 will be published by Springer-Verlag in Springer Series in Computational Neuroscience. This selection will be done by the Conference Chair and Program Co-chairs, among the papers actually presented at the conference, based on a rigorous review by the NEUROTECHNIX 2013 Program Committee members. 


\section{FOREWORD}

This book contains the proceedings of the 1st International Congress on Neurotechnology, Electronics and Informatics (NEUROTECHNIX 2013).

NEUROTECHNIX 2013 is co-sponsored by INSTICC - Institute for Systems and Technologies of Information, Control and Communication and MedinRes - Medical Information and Research, held in cooperation with Neurotech Network, Nansen Neuroscience Network, Associação Portuguesa de EEG e Neurofisiologia Clínica, Sociedade Portuguesa de Neurologia and Fp7 Project Enlightenment, and held in collaboration with Antibodies Online.

The Congress Technical Program includes oral presentations (full papers, short papers, and posters) organized around several topics such as: Neuromuscular Diseases, Parkinson Disease, Developmental Disorders, Dementia, Epilepsy, Sleep Disorders, Multiple Sclerosis, Neuroinfections, Brain Tumors, Stroke, Traumatic Brain Injuries, Cerebral Palsy, Spinal Cord Injury, Vision and Hearing Disorders. All full papers and short papers were included in the proceedings. The congress includes also several thematic sessions organized by specialized researchers: VirtRehab 2013 - Virtual and Augmented Reality Systems for Upper Limbs Rehabilitation; DeNeuro 2013 - Decoding the Neural Drive to Muscle through the Analysis of Motor Neuron Spike Trains; SensoryFusion 2013 - Sensory Fusion for Diagnostics and Neurorehabilitation; RoboAssist 2013 - Wearable Robotics for Motion Assistance and Rehabilitation; and BrainRehab 2013 - Brain-computer Interfaces and Brain Stimulation for Neurorehabilitation. A major aspect of the program is the inclusion of a set of four plenary keynote lectures given by internationally distinguished researchers, namely: Kevin Warwick (University of Reading, United Kingdom), François Hug (University of Queensland, Australia), Aldo Faisal (Imperial College London, United Kingdom) and Alexandre Castro-Caldas (Catholic University of Portugal, Portugal).

NEUROTECHNIX received submissions from 21 countries, in all continents. To evaluate each submission, a double blind paper review was performed by the Program Committee, whose members are highly qualified researchers in the NEUROTECHNIX topic areas. A post-congress Special Issue of the Springer Series in Computational Neuroscience is planned for publication of extended and revised versions of a restricted number of high quality papers presented during NEUROTECHNIX 2013. All papers presented at this congress will be available at the SciTePress Digital Library.

Congresses are also meeting places where collaboration projects can emerge from social contacts amongst the participants. Therefore, in order to promote the development of research and professional networks, the congress includes in its social program a Congress Social Event \& Banquet in the evening of September 20.

We would like to express our thanks to all participants. First of all to the authors, whose quality work is the essence of this congress; secondly to all members of the Program Committee and auxiliary reviewers, who helped us with their expertise and valuable time. We 
would also like to deeply thank the invited speakers for their excellent contribution in sharing their knowledge and vision. Finally, a word of appreciation for the hard work of the secretariat: organizing a congress of this level is a task that can only be achieved by the collaborative effort of a dedicated and highly capable team.

\author{
Ana Rita Londral \\ Universidade de Lisboa, Portugal
}

Pedro Encarnação

Universidade Católica Portuguesa, Portugal

Jose Luis Pons

Instituto de Automatica Industrial, Spain 


\section{Contents}

\section{INVITED SPEAKERS}

\section{KEYNOTE SPEAKERS}

The Disappearing Human-Machine Divide Kevin Warwick

Analysis of Muscle Coordination in Sports - Perspectives from Electromyography and Elastography François Hug

Breaking into your Brain with Neurotechnology

Aldo Faisal

The Working Brain - Windows to the Outside World

Alexandre Castro-Caldas

\section{PAPERS}

\section{Full Papers}

Robotic Grasp Initiation by Gaze Independent Brain-controlled Selection of Virtual Reality Objects Christoph Reichert, Matthias Kennel, Rudolf Kruse, Hans-Jochen Heinze, Ulrich Schmucker, Hermann Hinrichs and Jochem W. Rieger

Striving for Better and Earlier Movement Prediction by Postprocessing of Classification Scores Sirko Straube, Anett Seeland and David Feess

A Low Cost Platform based on FES and Muscle Synergies for Postural Control Research and Rehabilitation

D. Galeano, F. Brunetti, D. Torricelli, S. Piazza and J. L. Pons

\section{Short PAPERS}

Proposal of a P300-based BCI Speller using a Predictive Text System Ricardo Ron Angevin and Leandro da Silva-Sauer

Memory and Processing Efficient Formula for Moving Variance Calculation in EEG and EMG Signal Processing Mario Michael Krell, Marc Tabie, Hendrik Wöhrle and Elsa Andrea Kirchner

A Dataflow-based Mobile Brain Reading System on Chip with Supervised Online Calibration - For Usage without Acquisition of Training Data

Hendrik Woehrle, Johannes Teiwes, Mario Michael Krell, Elsa Andrea Kirchner and Frank Kirchner

A Method to Detect Keystrokes using Accelerometry to Quantify Typing Rate and Monitor Neurodegenerative Progression

Ana Londral, Mafalda Câmara, Hugo Gamboa, Mamede de Carvalho, Anabela Pinto and Luís Azevedo

Comparison of Neural Networks for Prediction of Sleep Apnea

Yashar Maali and Adel Al-Jumaily 


\section{SPECIAL SESSION ON VIRTUAL AND AUGMENTED REALITY SYSTEMS FOR UPPER LIMBS REHABILITATION}

\section{Full Papers}

Virtual Arm Representation and Multimodal Monitoring for the Upper Limb Robot Assisted Teletherapy

Gorka Epelde, Xabier Valencia, Aitor Ardanza, Elsa Fanchon, Alessandro De Mauro,

Francisco Molina Rueda, Eduardo Carrasco and Shabs Rajasekharan

Clinical, Functional and Kinematic Correlations using the Virtual Reality System Toyra ${ }^{\circledR}$ as Upper Limb Rehabilitation Tool in People with Spinal Cord Injury

Iris Dimbwadyo-Terrer, Fernando Trincado-Alonso, Ana de los Reyes-Guzmán, Alberto Bernal-Sahún, Patricia López-Monteagudo, Begoña Polonio-López and Ángel Gil-Agudo

Rehabilitation for Children while Playing with a Robotic Assistant in a Serious Game

L. V. Calderita, P. Bustos, C. Suárez Mejías, B. Ferrer González and A. Bandera

\section{ShORT PAPERS}

Illusion Approach for Upper Limb Motor Rehabilitation

Yee Mon Aung and Adel Al-Jumaily

New Developments in the Gesture Therapy Platform - Past, Present and Future of our Research Felipe Orihuela-Espina, Paloma Álvarez-Cardenas, Lorena Palafox, Israel Sánchez-Villavicencio, Alberto L. Morán, Jorge Hérnandez-Franco and Luis Enrique Sucar

\section{SPECIAL SESSION ON DECODING THE NEURAL DRIVE TO MUSCLE THROUGH THE ANALYSIS OF MOTOR NEURON SPIKE TRAINS}

\section{ShORT PAPERS}

Multi Channel Surface EMG - Detection and Conditioning

M. Gazzoni and U. Barone

On the Impact of Pathological Tremor Intensity on Noninvasive Characterization of Motor Unit Discharge Properties

Petra Povalej Bržan, Vojko Glaser, Simon Zelič, Juan Álvaro Gallego, Juan Pablo Romero Muñoz and Aleš Holobar

Motor Unit Properties and Underlying Determinants in Pathological Tremor

J. A. Gallego, J. L. Dideriksen, A. Holobar, J. P. Romero, J. L. Pons, E. Rocon and D. Farina

\section{SPECIAL SESSION ON SENSORY FUSION FOR DIAGNOSTICS AND NEUROREHABILITATION}

\section{Full Papers}

Assessment of Walker-assisted Human Interaction from LRF and Wearable Wireless Inertial Sensors

Maria Martins, Carlos Cifuentes, Arlindo Elias, Valmir Schneider, Anselmo Frizera and Cristina Santos

Human-like Sensor Fusion Mechanisms in a Postural Control Robot

Georg Hettich, Vittorio Lippi and Thomas Mergner 
Assessment of the Suitability of the Motorized Ankle-Foot Orthosis as a Diagnostic and Rehabilitation Tool for Gait

Guillermo Asín, Filipe A. Barroso, Juan C. Moreno and José L. Pons

Error Augmented Robotic Rehabilitation of the Upper Limb - A Review

Aris C. Alexoulis-Chrysovergis, Andrew Weightman, Emma Hodson-Tole and Frederik J. A. Deconinck

\section{SPECIAL SESSION ON WEARABLE ROBOTICS FOR MOTION ASSISTANCE AND REHABILITATION}

\section{Full Papers}

A Double-differential Actuation for an Assistive Hip Orthosis - Specificities and Implementation Jeremy Olivier, Mohamed Bouri and Hannes Bleuler

Feasibility of Hybrid Gait Training with Kinesis Overground Robot for Persons with incomplete Spinal Cord Injury

Antonio J. del-Ama, Ángel Gil-Agudo, José L. Pons and Juan C. Moreno

A Light-weight Exoskeleton for Hip Flexion-extension Assistance

Francesco Giovacchini, Matteo Fantozzi, Mariele Peroni, Matteo Moisè, Marco Cempini, Mario Cortese,

Dirk Lefeber, Maria Chiara Carrozza and Nicola Vitiello

Human Motion Assistance using Walking-aid Robot and Wearable Sensors

Jian Huang, Wenxia Xu, Zhen Shu and Samer Mohammed

Human-based Lower Limb Movement Assistance and Rehabilitation through an Actuated Orthosis Samer Mohammed, Hala Rifai, Walid Hassani and Yacine Amirat

\section{ShORT PAPERS}

Humanoids Meet Rehabilitation - Concept and Potential

Diego Torricelli and Jose L. Pons

Real-time Classification of Finger Movements using Two-channel Surface Electromyography Khairul Anam and Adel Al-Jumaily

Ankle-Knee Prosthesis with Powered Ankle and Energy Transfer - Development of the CYBERLEGs Alpha-Prototype

Louis Flynn, Joost Geeroms, Rene Jimenez-Fabian, Bram Vanderborght, Nicola Vitiello and Dirk Lefeber

\section{SPECIAL SESSION ON BRAIN-COMPUTER INTERFACES AND BRAIN STIMULATION FOR NEUROREHABILITATION}

\section{Full Paper}

Efficiency of SSVEF Recognition from the Magnetoencephalogram - A Comparison of Spectral Feature Classification and CCA-based Prediction

Christoph Reichert, Matthias Kennel, Rudolf Kruse, Hermann Hinrichs and Jochem W. Rieger 


\section{Short Papers}

Dynamics of a Stimulation-evoked ECoG Potential During Stroke Rehabilitation - A Case Study Armin Walter, Georgios Naros, Martin Spüler, Wolfgang Rosenstiel, Alireza Gharabaghi and Martin Bogdan

A Serious Game Application using EEG-based Brain Computer Interface

Francisco José Perales and Esperança Amengual

Monitoring Depth of Hypnosis under Propofol General Anaesthesia - Granger Causality and Hidden Markov Models

Nicoletta Nicolaou and Julius Georgiou

AUTHOR INDEX

263 